\title{
LAS ANTOCIANIDINAS COMO INDICADORES: \\ UNA EXPERIENCIA DE APRENDIZAJE \\ ALREDEDOR DEL PROCESO \\ DE NEUTRALIZACIÓN
}

Rafael Humberto Ramírez Gil

Universidad Pedagógica Nacional.

Luis Orlando Correa Monterrosa

Universidad de Córdoba.

\begin{abstract}
A man is in the center of an ecosystem, everything that rounds him is potentially use full. Man uses plants as a natural resource for survival as well as for learning of scientific concepts, among others. In the current work antocianidines present in summer flowers (Bouganviliea Glabra) and egg plant peels (Solanum Melongena) were used as $\mathrm{pH}$ indicators for strong acid-strong base neutralization reactions, replacing bromotimol. As an alternative for the work, students were involved in an active and participating process for solving problems through research based on F Coit Butler's model, David Ausubel's fundamental principle on educational psychology and research activities.

The methodology used includes performing a pre-test to determine the students' knowledge level, followed by four learning activities that lead to the solution, through research, of a real life problem, the concept normality concept, the plants active principles isolation such as the antocianidines, ant the neutralization concept.
\end{abstract}

\section{RESUMEN}

Siendo el hombre el epicentro de un ecosistema tiene a su disposición todo lo de su entorno, utiliza las plantas como recurso natural para su subsistencia y aprendizaje de conceptos científicos entre otros.

En el presente trabajo se utilizaron las antianidinas presentes en las flores de verano (Bougainvillea glabra) y cortezas de berenjena (Salanum melongena) como indicadores de $\mathrm{pH}$ en reacciones de neutralización ácido fuerte-base fuerte, reemplazado al bromotimol.

Como alternativa de trabajo se involucró al estudiante en un proceso activo participativo en la solución de problemas por investigación tomando como base teórica el modelo de F. Coit Butler, el principio fundamental de psicología educativa de David Ausubel y la actividad investigativa.

Como metodología se elaboró un pre-test para determinar preconceptos de los alumnos, desarrollando posteriormente cuatro actividades de aprendizaje que dieron solución por 
investigación a un problema de la vida diaria, al concepto de normalidad, al aislamiento de principios activos de las plantas como las antocianidinas y al concepto de neutralización.

\section{INTRODUCCIÓN}

La Fitoquímica, es la parte de la química que estudia los principios activos de los Vegetales y por ende la relación de estos con su entorno.

Para Walter Sheare (1987): "Desde que se reconoció que la Tierra era una esfera, los científicos han hecho muchos esfuerzos tratando de reducir esa esfera a proporciones que sean más dóciles a sus técnicas analíticas. Esto ha conducido al estudio de "esferas" conceptuales, incluyendo la atmósfera (la parte gaseosa), la litosfera (la parte sólida), la hidrosfera (la parte acuosa) y la biosfera (parte viviente).

Rápidamente se está acercando el momento en que será esencial, conceptualmente, integrar el conocimiento que se ha obtenido a través de los siglos, sobre cada uno de éstos componentes, para entender mejor cómo funciona el sistema total de la tierra".

Para entender esta interacción de "esferas" conceptuales entre sí e influenciadas por la acción del hombre, James $\mathrm{E}$. Lovelock ha propuesto el concepto de una ciencia llamada GEOFISIOLOGIA, como lo expone en el periódico editado por las Naciones Unidas. Foro de Desarrollo (F.D. Vol. XIII, No. 6 pág. 3 y No. 7 págs. 5 y 6 de 1985), en donde la interdependencia entre el hombre, los animales y las plantas, comparten con él, el agua, el aire y el suelo de una determinada área, manteniéndose un frágil y complementario equilibrio ecológico que solo la cooperación generosa entre los individuos y los pueblos podrá asegurar que la vida continúe.

El hombre es el epicentro de un ecosistema y de todo proceso científico, psicológico, filosófico, sociológico, pedagógico o cualquier otro que se suceda en su medio. Es el actor principal de su hábitat y tiene a su disposición los ecosistemas del mundo para suplir sus necesidades.

El estudio del medio ambiente se puede enfocar, desde el punto de vista conceptual, como un triángulo equilátero en cuyo centro está el hombre como epicentro de todo proceso, rodeado de las "esferas' conceptuales, en donde por acción de la energía solar se originan todos los cambios fisicoquímicos que se suceden en nuestro planeta tierra y que son estudiados por las distintas disciplinas conocidas por expertos como física, química, biología, geología, entre otras.

Existe una interrelación muy estrecha de las plantas con el resto de los componentes del ecosistema. No es del caso entrar a analizar a profundidad la importancia que tienen los suelos, el agua, la atmósfera, los animales y, desde luego, las radiaciones sobre las plantas; pero si debemos anotar que estos elementos, además de otros, constituyen factor esencial en la formación de miles de sustancias que se forman en las diferentes partes de los vegetales dando a éstos el interés particular que depende de la clase de principio activo que se forme; unas plantas tienen interés medicinal, otras son tóxicas, otras industriales y muchas son alimenticias, acorde con la fisiología vegetal, dando origen a los productos naturales vegetales.

La investigación en cualquier aspecto del ecosistema natural, constituye material valioso para la aprehensión significativa de conceptos, basada en el trabajo individual del alumno, que requieren "la concreción de los problemas a investigar y la explicitación de 
las ideas previas sobre los mismos, la elaboración y selección de hipótesis de trabajo, el diseño y aplicación de instrumentos de investigación, y la elaboración de conclusiones y su comunicación", L.M. del Carmen (1988).

Hemos tomado las plantas como punto de partida para los trabajos de investigación con los estudiantes de Licenciatura en Química y el desarrollo de tesis con los estudiantes de "Maestría en Docencia de la Química" lográndose varios objetivos como los de aprender a utilizar los recursos del medio como instrumento para lograr una mejor docencia a nivel de educación media y universitaria en el área de química. Aprender a manejar técnicas para recolección y clasificación de plantas objeto de estudio; extracción, separación y purificación de los componentes químicos y, determinación de estructuras.

El análisis fitoquimico motiva al investigador, quizá por que es uno de los campos más gratos de la química; se ven los resultados y permite formular hipótesis, explorar, plantear y definir nuevos problemas; facilitan la explicación de las propias ideas, pasando de lo natural a lo abstracto; le permite construir nuevas ideas que lo proyectan a generar nuevos conocimientos.

La concepción del medio no debe ser empírica, esto es, "coherente con la posición filosófica del positivismo lógico que defiende que las teorías científicas son inferidas, a partir de datos por medio de una lógica inductiva", Cawthron y Rowell (1978). Teoría que ha sido rebatida por filósofos como Kuhn (1970) y Toulmin (1972) quienes defienden que las teorías son invenciones de la mente humana que determinan, qué datos se recogen y cómo se interpretan.

El aprendizaje a partir del medio debe proporcionar al alumno oportunidad para desarrollar valores y actitudes; "debe hacerse sobre el medio ya favor del medio" sociocultural y socionatural, con rigurosidad científica.

Desde el punto de vista pedagógico, la fitoquímica se puede trabajar con diferentes modelos siendo los más desarrollados el constructivista de Ausubel y el de solución de problemas por investigación como el de $\mathrm{F}$. Coit Butler, modelo éste que asume, que el aprendizaje progresa a través de eventos jerárquicos tanto en orden como en función formando un todo dinámico.

A la luz del marco teórico de los modelos mencionados, particularmente del modelo de enseñanza-aprendizaje de las ciencias de F. Coit Butler (4), se desarrolló el trabajo "LAS ANTOCIANINAS COMO INDICADORES: UNA EXPERIENCIA DE APRENDIZAJE ALREDEDOR DEL PROCESO DE NEUTRALIZACIÓN", trabajo en el que se propone una alternativa para involucrar al estudiante en un proceso activo participativo en la solución de problemas por investigación, bajo los principios de la psicología activa de David Ausubel(1). Se busca materializar el aprendizaje del concepto de neutralización propiciando situaciones de participación de los estudiantes en la solución, por investigación, de un problema de neutralización, usando antocianidinas como indicadores.

Las antocianidinas son pigmentos hidrosolubles presentes en flores, cortezas, hojas y raíces de ciertas especies vegetales(S), cambian de color de acuerdo al pH del medio. Su rango de viraje es similar al del azul de bromotimol, lo que las habilita para competir con éste indicador. Como muestra natural de la cual los estudiantes obtienen las antocianidinas, se usaron flores de verano y cortezas de berenjenas. 
Según Ausubel(1) la esencia del aprendizaje significativo reside en que ideas expresadas simbólicamente, son relacionadas de modo no arbitrario, de manera sustancial con lo que el alumno ya sabe: especialmente con algún aspecto esencial de su estructura de conocimiento.

El modelo de Butler(4), asume que el aprendizaje progresa a través de una serie básica de siete escenas o eventos que son jerárquicos tanto en orden como en función, y que tomados juntos forman un todo dinámico. Estas escenas son: Situación, motivación, organización, aplicación, evaluación, repetición y generalización. Partiendo de que las situaciones son cambiantes. Butler considera que el proceso de aprendizaje se desarrolla en forma circular ascendente a partir de la percepción de una situación, para que finalmente, el estudiante traduzca lo que aprendió a partir de éste proceso, a fin de acomodarse a una nueva situación para empezar de nuevo porque su situación ha cambiado, tanto interna como externamente. (Ver figura 1).

Raúl Quintero Calderón (10) en su trabajo de grado usó extractos acuosos de flores comunes de clima frío (Sabana de Bogotá) para probar el comportamiento de los pigmentos hidrosolubles (antocianinas frente a soluciones ácidas y básicas. Inés Bernal Ramírez(3), trabajó con un extracto alcohólico-ácido, determinando los intervalos de viraje y los pka. No advierte posibles interferencias debidas a la presencia de otros pigmentos hidrosolubles (antoxantinas) o iones metálicos pesados. En los anteriores trabajos no se aíslan antocianidinas ni se aplica una estrategia pedagógica para usar éstos pigmentos como indicadores del punto de equivalencia en el aprendizaje del concepto de neutralización. De donde el presente estudio viene a ser un complemento de aquellos.

En su operacionalización, se aplicó el pre-test para indagar por el estado conceptual previo del alumno en relación con su actitud hacia la investigación, interés por la química, razonamiento hipotético deductivo y conceptualización sobre neutralización ácido-base. Se desarrollaron 4 actividades de aprendizaje orientadas según los fundamentos teóricos aquí establecidos (Teoría de Ausubel, modelo de Butler y de nuevo el test (post-test) para observar el cambio conceptual del alumno. Se aclara que en éste trabajo no se usó grupo control por tratarse de una investigación semi-experimental (10).

\section{OBJETIVO}

El objetivo central del estudio, es demostrar que conduciendo el proceso de enseñanza-aprendizaje según el modelo de Butler(4) el principio fundamental de la psicología de Ausubel(1) y la actividad investigativa(7), se logra el aprendizaje significativo. Se pretende materializar el aprendizaje del concepto de neutralización propiciando situaciones de participación de los estudiantes en la solución por investigación de un problema de neutralización usando antocianidinas como indicadores del punto de equivalencia.

\section{PROBLEMA}

En el proceso educativo, una de las últimas tendencias que buscan cambiar el aprendizaje memorístico y repetitivo, es el aprendizaje significativo propuesto por David Ausubel(1), fundamentados en la psicología educativa de éste autor, en el modelo de Coit Butler para la enseñanza-aprendizaje de las ciencias naturales(4), en la actividad 
investigativa(7) y en la legislación nacional sobre educación como la Ley 115, nos hemos planteado interrogantes de que si a partir de los recursos naturales podremos mejorar el aprendizaje de la química en los alumnos de educación media y superior habiendo llevado a la práctica investigaciones como la que nos ocupa en donde nos preguntamos si un grupo de alumnos de undécimo grado de educación media vocacional del Colegio de la Sagrada Familia de Montería aprenderá significativamente el concepto de neutralización 


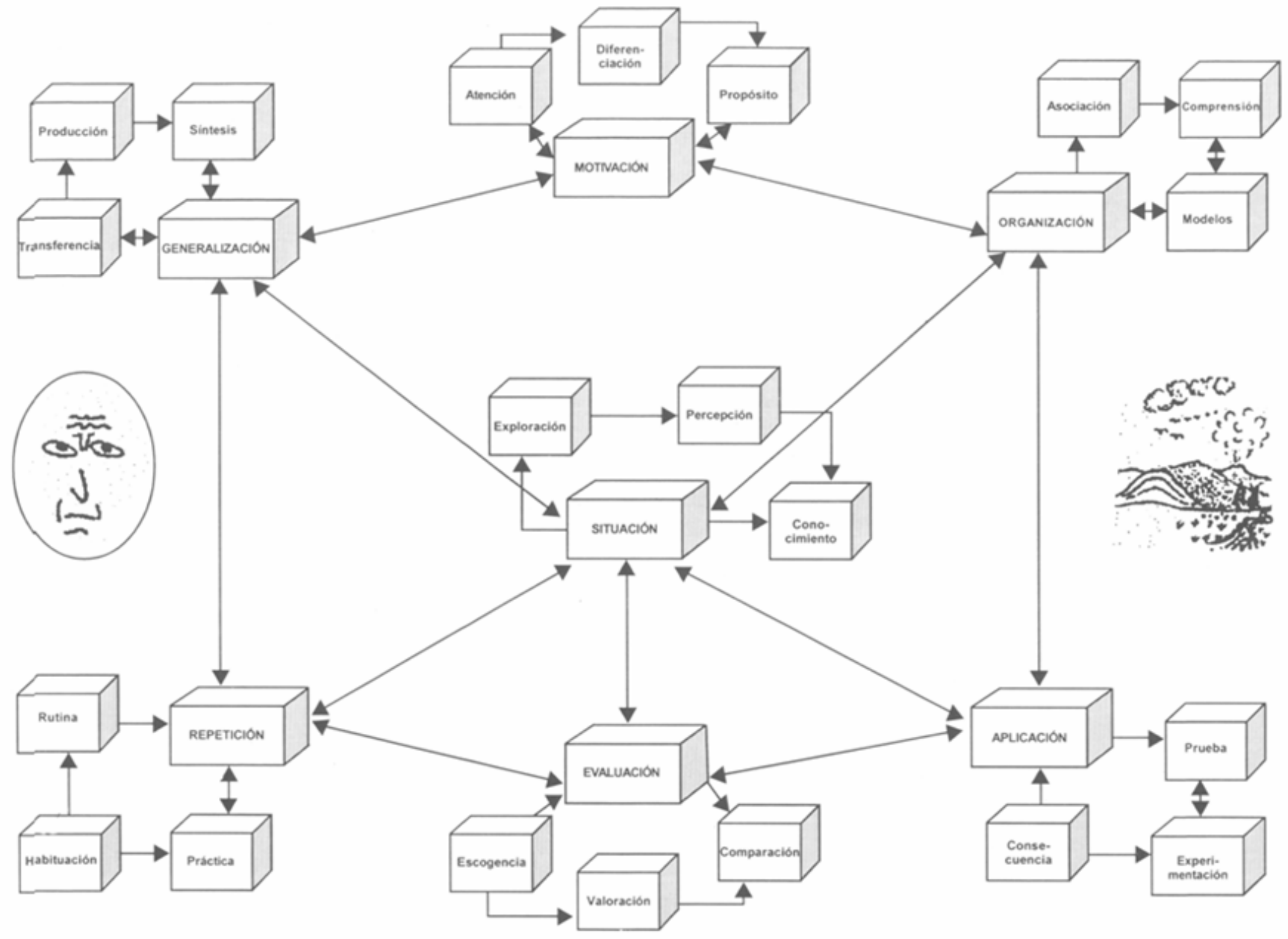

FIGURA 1. Los siete factores y escenas principales del aprendizaje (con actividad de aprendizaje, proceso cognitivo y resultado de cada uno. Modelo propuesto por F. Coit Butler. Adaptación del esquema publicado 
usando antocianidinas como indicadores extraídos de flores de verano y berenjenas. La respuesta ha sido positiva puesto que los resultados de la investigación demostraron que el proceso enseñanza-aprendizaje conducido de tal manera que el estudiante actúe como lo hace un investigador en ciencias, asegura un aprendizaje significativo y puede afirmar que la investigación es un proceso pedagógico.

\section{MARCO TEÓRICO}

En el modelo de Butler(4) se asume que el aprendizaje progresa a través de una serie de siete escenas o eventos que son jerárquicos tanto en orden como en función y que tomados juntos, forman un todo dinámico. Estas escenas son: (Ver Figura 2).

\section{Situación del alumno}

Según Butler, el aprendizaje tiene lugar dentro del contexto de una serie de circunstancias particulares del estudiante que surgen de una situación dada. Afirma que las etapas para el desarrollo del conocimiento de la situación (realización de un experimento por ejemplo), involucran previsión, exploración y percepción de elementos internos propios del individuo como intereses, actitudes, valores, hábitos y otros; o externos como instrumentos, reactivos, materiales de estudio y otros, que rodean al estudiante y que afectan el aprendizaje del nuevo conocimiento.

La previsión, exploración y percepción se evidencian en nuestro estudio cuando el alumno propone un diseño experimental para poner a prueba la hipótesis inicial de trabajo.

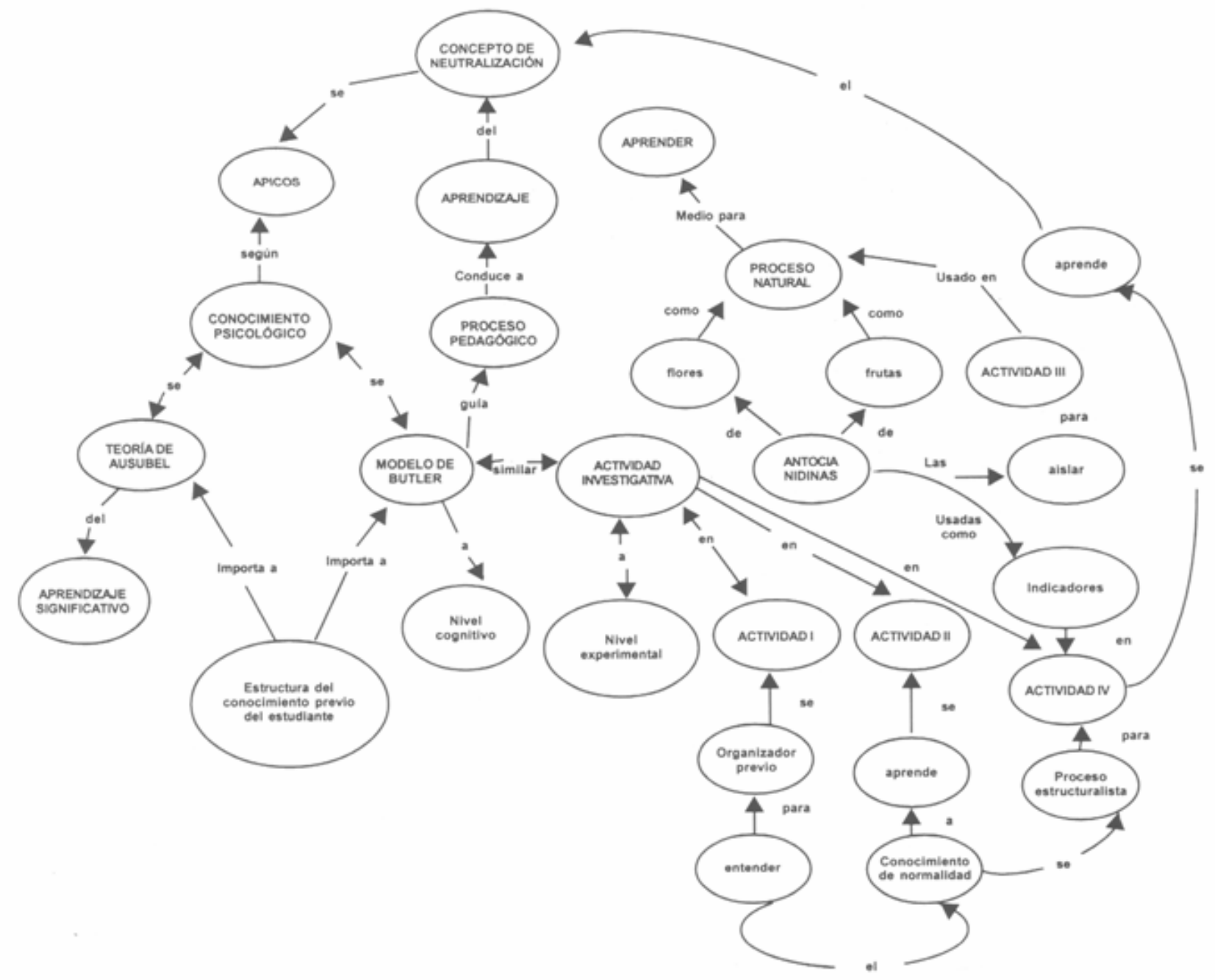


FIGURA 2. Mapa conceptual que reconcilia las tres teorías base del estudio: Teoría de Ausubel, Modelo de Butlery actividad investigativa.

\section{Motivación}

Se logra cuando el estudiante tenga la necesidad de aprender despertando en él expectativas de lo que se va a realizar (el diseño de un experimento), diferenciado entre lo importante y lo no importante, lo significativo. Esta estrategia permite centrar la atención a lo que se quiere enseñar. En química por ejemplo hay varias formas de expresión de concentración: molaridad, modalidad, porcentaje en peso por volumen, porcentaje en peso por peso, normalidad etc., de las escuelas para nuestro propósito de estudio, solo interesa la de normalidad y porcentaje en peso por peso.

\section{Organización de la información}

Cumplidas las etapas anteriores, según Butler se proporciona cierto grado de comprensión. Esta escena la vive el estudiante cuando el profesor solicita al grupo de investigación, elaborar un marco teórico de referencia que sustente la formulación de la hipótesis y el diseño experimental.

\section{Aplicación}

El profesor debe buscar un comprometimiento del alumno a través de una actividad responsable y continua. En nuestro trabajo éste compromiso lo adquiere el estudiante cuando el profesor le encomienda la tarea de encontrar una solución por investigación, al problema planteado.

Según Butler(4) los conceptos abstractos son más difíciles de aprender cuando no se concretizan poniéndolos a prueba. Dice además que el estudiante no puede aprender un proceso o procedimiento nuevamente leyendo acerca de él.

\section{Evaluación}

Butler dice que para la evaluación se debe seguir una retroalimentación selectiva, a fin de proveer las bases para la comparación de los resultados reales con los esperados. Se compara la respuesta con el conocimiento previo del estudiante y la experiencia que pueda proporcionar toda la retroalimentación requerida.

\section{Repetición}

No se refiere a la recitación memorística de proposiciones o conceptos extraídos de textos. Se trata de otra escena del modelo de Butler, considerada como una práctica y/o uso continuado de las bases para la habituación de los nuevos conocimientos y habilidades. Si el estudiante aprende significativamente conceptos, principios y procesos, la práctica repetitiva se reducirá al mínimo. La retención a largo plazo de lo aprendido significativamente, se conservará en gran parte, por ser conectado a la estructura cognitiva que ha desarrollado en el alumno. 


\section{Generalizaciones}

Para Butler (1985), la última nieta el proceso enseñanza-aprendizaje no es solamente que el estudiante adquiera conocimiento y habilidades, sino que éste pueda transferirlos más allá del aula de clase en la cual fueron aprendidos haciendo GENERALIZACIONES tendientes a aplicar las propuestas metodológicas desarrolladas por ellos mismos. En esta última escena se hacen inferencias y se establecen reglas para manejar la situación, considerando ésta como cambiante. Sintetizar dos o más ideas no es simplemente un proceso aditivo y lineal. En vez de ello es geométricamente progresivo y circular, ya que involucra interacciones de redes asociativas. (Ver Figura 1).

Como podemos ver, el modelo de Butler establece un paralelo entre el proceso de aprendizaje y el progreso de la ciencia a través de la actividad investigativa, partiendo de una situación inicial para llegar a un estado de generalización.

También es importante, no descuidar el modelo de aprendizaje por investigación llevando a los estudiantes a diseñar experimentos, formular hipótesis, establecer una metodología y estrategias que permitan analizar con rigor los resultados.

Los planteamientos teóricos de David Ausubel, de Coit Butler y los del modelo de aprendizaje por investigación, constituyen la base teórica del presente estudio y que se resumen en la figura 2, mapa conceptual que concilia las tres teorías apuntando el aprendizaje del concepto neutralización.

Podemos pensar en una hipótesis general y una particular; en la primera creemos que si el proceso enseñanza-aprendizaje se orienta de modo que el estudiante actúe como lo hace un investigador en ciencias naturales (V.I), se despertará en él una actitud hacia la investigación y un interés por la química (V.D). Además, si la actividad investigativa produce cambios conceptuales en el estudiante (V.I), la investigación es un proceso pedagógico (V.D).

Como hipótesis particular se considera que siguiendo las estrategias del modelo de Butier, el principio fundamental de la psicología educativa de Ausubel, y la actividad investigativa (VI), los alumnos aprenderán el concepto de neutralización, usando antocianidinas como indicadores (V.D).

\section{METODOLOGÍA}

Como investigación semiexperimental, en el presente estudio se utilizó un grupo experimental con pre-test y post-test(10).

Participaron en el experimento 24 alumnos de undécimo grado de educación media vocacional del Colegio de la Sagrada Familia de Montería-Córdoba, con una duración de 28 sesiones de 90 minutos cada una.

Los conceptos tratados en la fase experimental del estudio fueron: Relación número de alumnas/área de aula, normalidad, solución, ácido, base, equivalente gramo, pH, indicadores, antocianidinas, punto de equivalencia, punto final, titulación y neutralización. Para conocer el estado conceptual previo (situación inicial) del alumno, en relación con los 
conceptos mencionados, se aplicó un test (pretest) destinado a medir cuatro categorías de respuesta: Interés por la química; actitud investigativa, razonamiento hipotético deductivo y conceptualización sobre neutralización.

Las preguntas fueron elaboradas con una "entradilla" (Carrizosa, 1990) para ubicar al alumno en la temática. Para cada una de las cuatro categorías de respuesta, se utilizaron matrices de evaluación. La mayoría de las preguntas que las sustentan son de selección múltiple en las que se hace un planteamiento de partida, a modo de interrogación o afirmación, y se ofrecen varias alternativas de respuesta.

Los resultados del pre-test, mostraron deficiencias en el razonamiento hipotéticodeductivo y niveles medio- bajo en las demás categorías (Véase Tabla 1 y Figura 3). Partiendo de esta situación se realizó una actividad que sirviera de organizador previo(1) buscando un puente cognitivo entre lo que el alumno ya sabe (situación de la vida cotidiana o concreta) y el concepto de normalidad (abstracto) el concepto de normalidad, a su vez, se subordina al concepto de neutralización más inclusivo que se pretende enseñar.

Como parte de la metodología se desarrollaron cuatro ACTIVIDADES DE APRENDIZAJE básicas que conducen al aprendizaje del concepto de neutralización. Las actividades uno, dos y cuatro se inician con el planteamiento de un problema que lo resuelven en grupo, según la metodología de la investigación científica, y la dirección del profesor. En la actividad tres, los estudiantes siguiendo el método proporcionado por el profesor, obtienen las antocianidinas a partir de flores de verano (Bougainvillea glabra) y berenjenas (Solanum melongena), para su utilización como indicadores del punto de equivalencia de una reacción de neutralización.

ACTIVIDAD 1: SOLUCIÓN, POR INVESTIGACIÓN, DE UN PROBLEMA DE LA VIDA COTIDIANA. Esta actividad sirvió de organizador previo(1). Se quiso establecer un puente cognitivo entre un concepto extraído de la vida cotidiana: Relación: "número de alumnos/áreas de aulas" y concepto abstracto: "número de equivalentes — gramo/litro de solución (normalidad)": ambas relaciones como expresiones de concentración.

El problema se plantea en forma de interrogante mediante proposiciones que relacionan conceptos familiares al estudiante con significado potencial.

El problema se planteó así: “¿Es la relación número de alumnas/sobre áreas de aulas del Colegio de la Sagrada Familia, adecuada para la docencia?

Para dar solución al problema se asumió que 15 a 20 estudiantes por $30 \mathrm{~m}^{2}$ de aula es una relación (concentración) apropiada para la docencia. Con base a ésta consideración cada grupo formuló una hipótesis, diseñó un experimento, operacionalizó el diseño, discutió los resultados confrontándolos con la hipótesis inicial del trabajo y los objetivos trazados, sacó unas conclusiones y rindió un informe. 


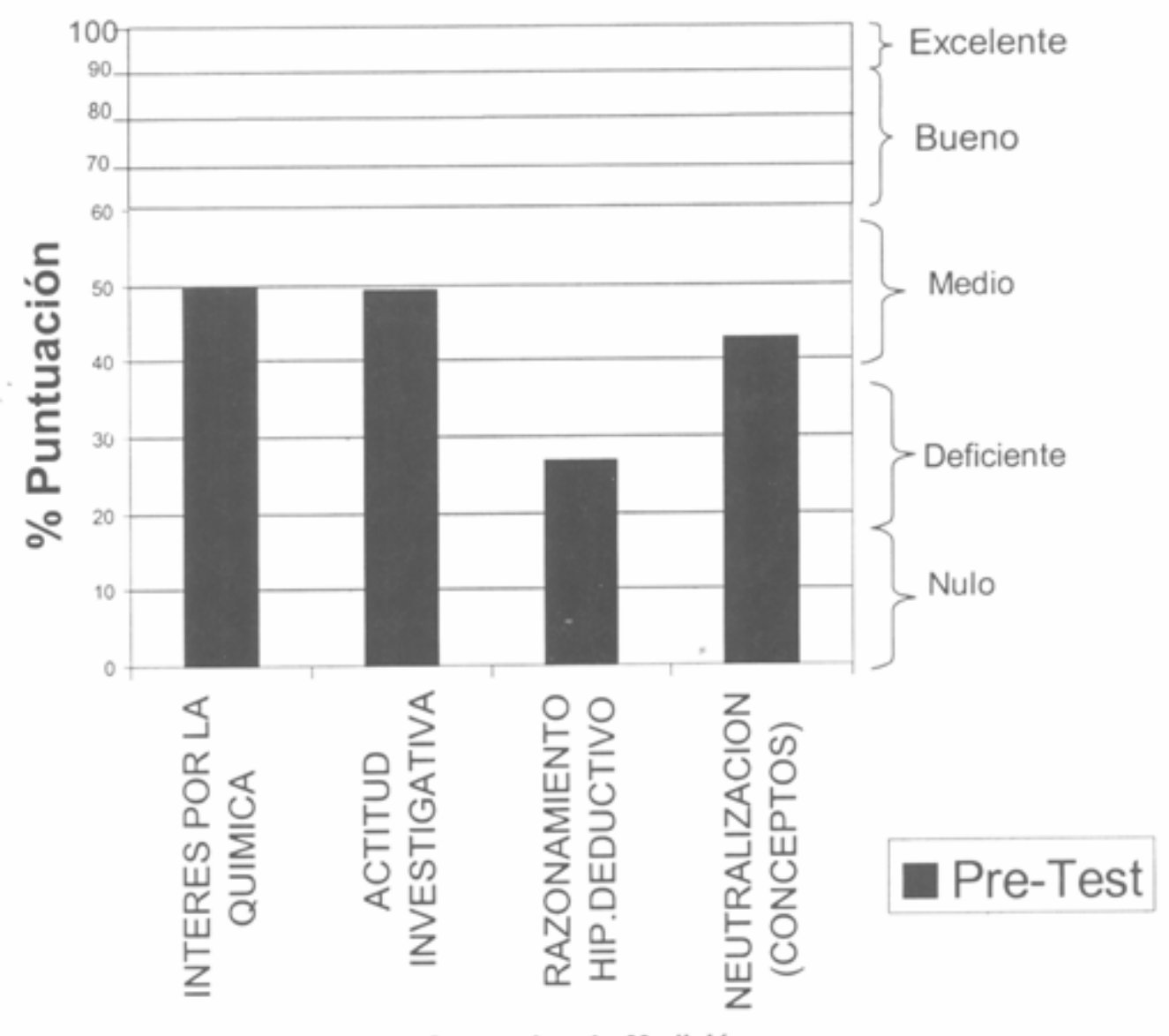

Categorías de Medición

FIGURA 3. Día grama de columna de los resultados del pre-test, aplicado a 22 alumnas de undécimo grado de Educación Media Vocacional del Colegio de la Sagrada Familia de Montería. 
TABLA 1

UNIVERSIDAD PEDAGÓGICA NACIONAL

RESULTADOS DEL POST-TEST APLICADO A 22 ALUMNAS DE UNDÉCIMO GRADO DE EDUCACIÓN MEDIA VOCACIONAL DEL COLEGIO DE LA SAGRADA FAMILIA DE MONTERÍA

\begin{tabular}{|c|c|c|c|c|c|c|c|c|}
\hline \multicolumn{9}{|c|}{ CATEGORIAS DE EVALUACION } \\
\hline & \multicolumn{2}{|c|}{1} & \multicolumn{2}{|c|}{2} & \multicolumn{2}{|c|}{3} & \multicolumn{2}{|c|}{4} \\
\hline SUJETO & \multicolumn{2}{|c|}{ INTERES POR LA QUÍMICA } & \multicolumn{2}{|c|}{ ACTITUD INVESTIGATIVA } & \multicolumn{2}{|c|}{ RAZONAMIENTO HIP. DECUCTIVO } & \multicolumn{2}{|c|}{ NEUTRALIZACIÓN CONCEPTOS } \\
\hline No & $\%$ Punt & Nivel & $\%$ Punt & Nivel & $\%$ Punt & Nivel & $\%$ Punt & Nivel \\
\hline 1 & 49.99 & Medio & 40.46 & Medio-I & 33.33 & Defic. & 21.65 & Defic. \\
\hline 2 & 49.99 & Medio & 38.07 & Defic. & 66.66 & Bueno & 34.98 & Defic. \\
\hline 3 & 66.66 & Bueno & 57.11 & Medio & 0 & Nulo & 36.64 & Defic. \\
\hline 4 & 49.99 & Medio & 66.64 & Medio & 0 & Nulo & 44.64 & Medio \\
\hline 5 & 49.99 & Medio & 36.88 & Defic. & 33.33 & Defic. & 38.31 & Defic. \\
\hline 6 & 66.66 & Bueno & 44.62 & Medio & 41.66 & Medio & 38.33 & Defic. \\
\hline 7 & 83.32 & Bueno & 61.88 & Bueno & 0 & Nulo & 39.97 & Defic. \\
\hline 8 & 49.99 & Medio & 52.95 & Medio & 33.33 & Defic. & 38.31 & Defic. \\
\hline 10 & 83.32 & Bueno & 57.12 & Medio & 66.66 & Bueno & 46.62 & Medio \\
\hline 11 & 66.66 & Bueno & 57.12 & Medio & 0 & Nulo & 43.29 & Medio \\
\hline 12 & 66.66 & Bueno & 59.50 & Medio & 41.66 & Medio-I & 63.29 & Bueno \\
\hline 13 & 33.33 & Defic. & 54.14 & Medio & 0 & Nulo & 38.30 & Defic. \\
\hline 15 & 66.66 & Bueno & 36.29 & Defic. & 66.66 & Bueno & 53.30 & Medio \\
\hline 16 & 83.32 & Bueno & 57.12 & Medio & 41.66 & Medio-I & 38.30 & Defic. \\
\hline 17 & 0 & Nulo & 58.31 & Medio & 33.33 & Defic. & 59.94 & Medio \\
\hline 18 & 66.66 & Bueno & 61.28 & Bueno-I & 33.33 & Defic. & 49.96 & Medio \\
\hline 19 & 33.33 & Nulo & 65.44 & Bueno & 0 & Nulo & 38.31 & Defic. \\
\hline 20 & 0 & Nulo & 10.00 & Defic. & 33.33 & Defic. & 68.27 & Bueno \\
\hline 21 & 0 & Nulo & 52.36 & Medio & 0 & Nulo & 36.64 & Defic. \\
\hline 22 & 33.33 & Defic. & 33.91 & Defic. & 33.33 & Defic. & 43.31 & Medio \\
\hline 23 & 33.33 & Defic. & 40.45 & Medio & 0 & Nulo & 34.98 & Defic. \\
\hline 24 & 66.66 & Bueno & 47.00 & medio & 33.33 & Defic. & 34.98 & Defic. \\
\hline
\end{tabular}


ACTIVIDAD II: SOLUCIÓN POR INVESTIGACIÓN DE UN PROBLEMA DE NORMALIDAD. El concepto de normalidad es un concepto subordinado del concepto de neutralización más inclusivo o supra ordenado (1). "número de equivalentes/litro de solución", el estudiante encuentra una relación de asociación con otra expresión de concentración ya conocida "número de alumnas/área de aula",

PROBLEMA: ¿Cómo preparar $500 \mathrm{ml}$ de una solución de $\mathrm{H}_{2} \mathrm{SO}_{4} 0.5 \mathrm{~N}$, a partir de $\mathrm{H}_{2} \mathrm{SO}_{4}$ grado analítico de $96 \%$ de pureza y densidad $1.84 \mathrm{~g} / \mathrm{ml}$ ?

HIPÓTESIS: Los primeros intentos de formulación de hipótesis fueron infructuosos, luego de la orientación del profesor, se concretaron algunas como la siguiente: "si tomamos siete mililitros de $\mathrm{H}_{2} \mathrm{SO}_{4}$ de $96 \%$ de pureza y densidad $1.84 \mathrm{~g} / \mathrm{ml}$, y lo diluimos con agua destilada hasta completar $500 \mathrm{ml}(\mathrm{VI})$ obtenemos una solución 0.5 Normal (V.D).

DISEÑO EXPERIMENTAL: Consistió en una propuesta metodológica que los estudiantes elaboraron tomando como patrón de dirección la hipótesis formulada. Esto permitió a los estudiantes familiarizarse con los nombres y el uso de instrumentos de medición y se comprometa en la investigación activa y la prueba de los conceptos, principio y procedimiento cuando se presenten.

Cada grupo preparó la solución de ácido y de base solicitada en el problema, siguiendo las directrices señaladas en el diseño experimental propuesto.

A manera de discusión sobre el desarrollo de ésta actividad, podemos decir que el aprendizaje del concepto de normalidad según esta metodología, no depende siempre del conocimiento de los resultados de una fuente externa. Esto es cierto cuando la respuesta o la acción, es guiada por la actividad investigativa. A veces no se tiene el resultado esperado, lo que desmotiva; se puede motivar estimulando el trabajo.

Como conclusión podemos decir que la actividad II permitió a los estudiantes aplicar los conceptos, principios o procesos en un contexto diferente que representa la generalización de lo aprendido. También se puede concluir que aprendieron el concepto de normalidad debido a la formulación de la hipótesis y su aplicación en la solución experimental del problema.

ACTIVIDAD III: AISLAMIENTO DE LAS ANTOCIANIDINAS. A partir de flores y frutos constituyó un factor de motivación. Los estudiantes tenían la creencia de que en las flores solo había sustancias aromáticas que se usaban para elaborar perfumes, cuando supieron que contenían antocianidinas que se utilizaban como indicadores de $\mathrm{pH}$, empezaron a diferenciar entre sus ideas previas y el nuevo conocimiento.

Con esta escena se pone de manifiesto que el nivel de atención está directamente relacionado con el nivel de afecto despertado por la percepción del estudiante de la situación natural. Con esto se buscó utilizar un recurso natural como medio para aprender química, concretamente aislar antocianidinas a partir de flores de verano y berenjenas para usarlas como indicadores en la solución de un problema de neutralización.

En la separación de las antocianidinas se siguió una técnica fitoquímica explicada por el profesor, proceso que duró tres semanas al cabo de las cuales se obtuvo el producto que fue utilizado en la actividad IV. 
ACTIVIDAD IV: SOLUCIÓN AL PROBLEMA DE NEUTRALIZACIÓN. MEDIANTE LAS ANTOCIANIDINAS. Nos preguntamos cómo diseñar y hacer un experimento para determinar el punto de equivalencia de una reacción de neutralización, usando antocianidinas como indicadores y predecir el volumen de $\mathrm{NaOH} 0.25 \mathrm{~N}$, necesario para

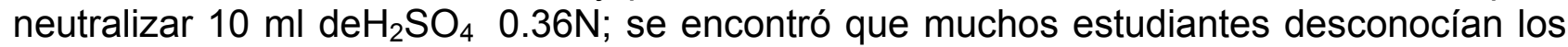
conceptos de punto de equivalencia y neutralización, generando acciones que permitieran relacionar los conceptos subordinados menos incluyentes para llegar a uno más incluyente (supra ordenado), generando hipótesis por parte de los alumnos como lo siguiente: "Si tomamos $10 \mathrm{ml} \mathrm{de} \mathrm{H}_{2} \mathrm{SO}_{4} 0.36 \mathrm{~N}$, y le agregamos $14.28 \mathrm{ml}$ de $\mathrm{NaOH} 0.25$ $\mathrm{N}$ logramos la neutralización. Agregando 5 gotas de antocianidina al ácido, éste se tornará rojizo y al agregar con la bureta la base, habrá un cambio de color (rojo-verde-amarillo) que nos indicará el punto de equivalencia".

Esta actividad y la anterior corresponden a lo que en el modelo Butler es la ORGANIZACIÓN, en donde se busca que los principios y conceptos generales sirvan de organizadores que proporcionen coherencia y significado a las áreas del conocimiento.

Al enfrentarse la tarea del aprendizaje, el estudiante tiene la necesidad de construir modelos organizados para dar significado a los contenidos; después se propone un diseño que incluya los instrumentos de medición en cuya operacionalización el estudiante se compromete activamente, aplicando y probando los nuevos conceptos, principios y procesos permitiendo familiarizarse con el nombre y uso de los instrumentos.

Los conceptos como los de normalidad, indicadores, punto de equivalencia etc., encontraron su aplicabilidad al determinar experimentalmente el volumen de $\mathrm{NaOH}$ requerido para neutralizar $10 \mathrm{ml}$ de $\mathrm{H}_{2} \mathrm{SO}_{4} 0.36 \mathrm{~N}$. El punto de equivalencia determinado con antocianidinas es comparado con el del azul de Bromotimol.

Esta escena corresponde a lo que en el modelo de Butler es la evaluación en donde cada grupo discute los resultados. Como conclusión podemos decir que el trabajo experimental es similar a la generalización que sigue a una observación dialéctica de los fenómenos, principios y procesos.

Para llegar a la generalización se confrontaron los resultados del experimento con la hipótesis inicial de trabajo y los objetivos propuestos.

\section{ANÁLISIS DE RESULTADOS}

La fase final del estudio fue la aplicación por segunda vez del test (post-test), cuyos resultados se resumen en tabla 2 y la figura 4 . 


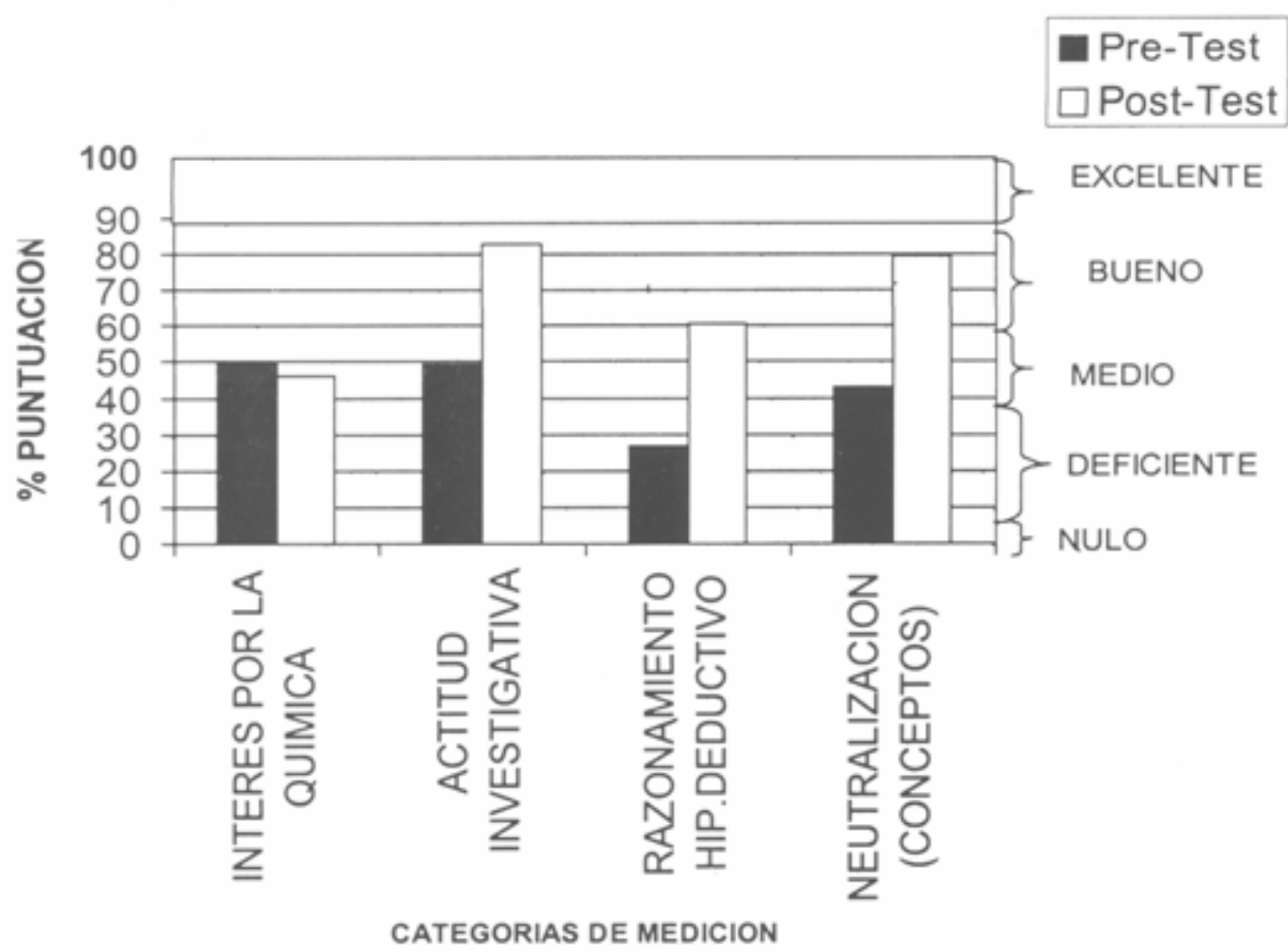

FIGURA 4. Diagrama comparativo de columnas de los resultados del pre-test y post-test, aplicado a 22 alumnas de undécimo grado de Educación Media Vocacional del Colegio de la Sagrada Familia de Montería, Córdoba. 
TABLA 2

RESULTADOS DEL POST-TEST APLICADO A 22 ALUMNAS DE UNDECIMO GRADO DE EDUCACION MEDIA VOCACIONAL DEL COLEGIO DE LA SAGRADA FAMILIA DE MONTERIA

\begin{tabular}{|c|c|c|c|c|c|c|c|c|}
\hline \multicolumn{9}{|c|}{ CATEGORIAS DE EVALUACION } \\
\hline \multirow{3}{*}{$\frac{\text { SUJETO }}{\mathbf{N}^{\circ}}$} & \multicolumn{2}{|c|}{1} & \multicolumn{2}{|c|}{2} & \multicolumn{2}{|c|}{3} & \multicolumn{2}{|c|}{4} \\
\hline & \multicolumn{2}{|c|}{ INTERES POR LA QUIMICA } & \multicolumn{2}{|c|}{ ACTITUD INVESTIGATIVA } & \multicolumn{2}{|c|}{$\begin{array}{c}\text { RAZONAMIENTO HIP } \\
\text { DEDUCTIVO }\end{array}$} & \multicolumn{2}{|c|}{$\begin{array}{l}\text { NEUTRALIZACION } \\
\text { (CONCEPTOS) }\end{array}$} \\
\hline & $\%$ Punt & Nivel & $\%$ Punt & Nivel & $\%$ Punt & Nivel & $\%$ Punt & Nivel \\
\hline 1 & 33.33 & Defic. & 86.87 & Bueno & 33.33 & Defic. & 74.93 & Bueno \\
\hline 2 & 100.00 & Excelente & 83.30 & Bueno & 66.66 & Bueno & 66.62 & Bueno \\
\hline 3 & 33.33 & Defic. & 91.63 & Excelente & 66.66 & Bueno & 74.94 & Bueno \\
\hline 4 & 49.99 & Medio & 84.49 & Bueno & 33.33 & Defic. & 81.59 & Bueno \\
\hline 5 & 33.33 & Defic. & 84.49 & Bueno & 33.33 & Defic. & 74.94 & Bueno \\
\hline 6 & 66.66 & Bueno & 86.63 & Bueno & 33.33 & Defic. & 79.93 & Bueno \\
\hline 7 & 0 & Nulo & 78.54 & Bueno & 33.33 & Defic. & 78.26 & Bueno \\
\hline 8 & 49.99 & Medio & 83.30 & Bueno & 33.33 & Defic. & 79.93 & Bueno \\
\hline 10 & 83.32 & Bueno & 83.30 & Bueno & 66.66 & Bueno & 96.57 & Excelente \\
\hline 11 & 83.32 & Bueno & 92.82 & Excelente & 33.33 & Defic. & 83.25 & Bueno \\
\hline 12 & 49.99 & Medio & 88.06 & Bueno & 100.00 & Excelente & 64.94 & Bueno \\
\hline 13 & 33.33 & Defic. & 89.52 & Bueno & 100.00 & Excelente & 93.24 & Excelente \\
\hline 15 & 0 & Bueno & 83.30 & Bueno & 33.33 & Defic. & 81.59 & Bueno \\
\hline 16 & 66.66 & Defic. & 47.59 & Medio & 33.33 & Defic. & 83.26 & Bueno \\
\hline 17 & 0 & Nulo & 82.70 & Bueno & 66.66 & Bueno & 74.93 & Bueno \\
\hline 18 & 66.66 & Bueno & 78.54 & Bueno & 100.00 & Excelente & 68.27 & Bueno \\
\hline 19 & 0 & Nulo & 86.87 & Bueno & 66.66 & Bueno & 83.26 & Bueno \\
\hline 20 & 0 & Nulo & 71.00 & Bueno & 100.00 & Excelente & 83.26 & Bueno \\
\hline 21 & 33.33 & Defic. & 74.97 & Bueno & 100.00 & Excelente & 84.92 & Bueno \\
\hline 22 & 66.66 & Bueno & 86.87 & Bueno & 66.66 & Bueno & 83.25 & Bueno \\
\hline 23 & 66.66 & Bueno & 91.63 & Excelente & 33.33 & \begin{tabular}{|l|} 
Defic. \\
\end{tabular} & 79.93 & Bueno \\
\hline 24 & 33.33 & Defic. & 86.87 & Bueno & 100.00 & Excelente & 83.25 & Bueno \\
\hline
\end{tabular}


Comparando los resultados del pretest con los del post-test se observó un aumento significativo en las categorías de respuestas dos y cuatro. La actitud investigativa pasó de un nivel medio-inferior con una puntuación de $49.48 \%$ a bueno superior con una puntuación de 82.87 resultado que valida la hipótesis general del estudio.

La conceptualización de neutralización pasó de medio inferior con puntuación de $42.84 \%$ a bueno superior con puntuación de $79.77 \%$ corroborando así la hipótesis particular del estudio.

No hubo progreso significativo en la categoría tres (razonamiento hipotético deductivo) quizá por no haber incluido el concepto de pH entre los subordinados.

El proceso no modificó las aspiraciones del estudiante ya señaladas en el pretest.

Comparando las desviaciones estándar de las categorías de respuestas dos y cuatro del pre-test y del post-test se observa una disminución de la misma.

\section{CONCLUSIONES}

- El análisis de los resultados demuestra que el proceso enseñanza-aprendizaje conducido de manera que el estudiante actúe como lo hace un investigador en ciencias, asegura un aprendizaje significativo y se puede asegurar que la investigación es un proceso pedagógico.

- Al considerar en éste estudio las estrategias-aprendizaje del modelo de Butler que se pudieron operacionalizar, el principio fundamental de la tecnología educativa de Ausubel y la actividad investígativa como variables independientes, los resultados de la evaluación final (post-test), indican que el aprendizaje fue significativo.

- Las antocianidinas extraídas de flores de verano (Bongainvillea glabra) y cortezas de berenjena (Solanum melongena) por sus propiedades de indicadores de $\mathrm{pH}$ pueden reemplazar el azul de Bromo-timol como indicador del punto de equivalencia en reacciones de neutralización ácido fuerte-base fuerte.

\section{BIBLIOGRAFÍA}

1. AUSUBEL D. Psicología Educativa. Un punto cognoscitivo. Trillas, 1976.

2. BARAHONA, A. y BARAHONA, F. Metodología de trabajos científicos. IPLER. Bogotá, 1976.

3. BERNAL DE RAMíREZ, I. Contribución al estudio de los pigmentos de algunas flores y frutos como indicadores ácido-base. Química e Industria V-14. Bogotá. 1988.

4. BUTLER F. Coit. The teaching learning process: A unified, interactive Model Educational Technology. V.25 (9) sept. (10) oct. (11) nov. 1985. 
5. CORREA M., LO. Propuesta de un método para el aislamiento de antocianidinas. Departamento de Química, Universidad Pedagógica Nacional, Bogotá 1990.

6. ESCALANTE ANGULO, C. Medición de actitudes. Fondo Nacional Universitario. Bogotá, 1990.

7. GIL PÉREZ D. Tres paradigmas básicos en la enseñanza de las ciencias. Enseñanza de las ciencias, 1983, pp. 26-33.

8. GAGLIARDI, R. Los conceptos estructurantes en el aprendizaje por investigación. Enseñanza de las Ciencias 4 (1), 30-35. 1986.

9. NORMAS COLOMBIANAS SOBRE DOCUMENTACIÓN DE TESIS DE GRADO. ICONTEC. Bogotá. 1987.

10. QUINTERO CALDERON R. Algunas flores comunes de clima frío como indicadores naturales. Tesis de grado. Departamento de Química, Universidad Pedagógica Nacional, Bogotá, 1988.

11. RAMíREZ GIL R.H. La Fitoquímica y su incidencia en la enseñanza de la Química. Departamento de Química. Universidad Pedagógica Nacional. Bogotá, 1990.

12. RODRIGUEZ A. W. Tecnología de la investigación experimental en educación. eeLcab. Lima. 